\title{
Artificial intelligence-enabled technologies and clinical decision making
}

\author{
Ryan Chan, Adam Morse
}

Artificial intelligence involves the theory, practice, and design of systems that exhibit functional human intelligence characteristics such as perception, problem solving, and planning. ${ }^{1}$ Further, artificial intelligence has been defined as the study of intelligent agents or agents that possess these human-like traits, and can interact with their environment through various sensory and input mechanisms to formulate an action in order to achieve a goal or outcome. ${ }^{2}$ These unique properties have gained a wide interest from interdisciplinary communities, which are investigating the potential applications of intelligent-enabled technologies. Within healthcare, there has been a progressive diffusion and integration of intelligent agent-enabled technologies across consumer populations (such as smart technologies) as well as in many health care organizations and settings (ie acute care, home care)., ${ }^{3,4}$ In addition, advanced technologies associated with intelligent agents that have the ability to formulate diagnosis and treatment plans have also been introduced into both the clinical setting and practice.

IBM Watson is a cognitive technology that leverages big data through screening extensive volumes of medical evidence and literature. ${ }^{5}$ It has the capability to assist with life sciences research and to rapidly formulate medical decisions with greater accuracy than physicians. ${ }^{5,6}$ Specifically, IBM Watson has been introduced to assist with decision making related to lung cancer treatment; this is based on its ability to synthesize and analyze data, including case scenarios and research evidence, and to formulate clinical decisions such as choice of treatments. ${ }^{7,8}$ Despite its promising capabilities, projects and partnerships with academic medical centres related to this lung cancer treatment program have been discontinued, as the treatment decisions provided by Watson were found to be inappropriate and not safe for patients. ${ }^{910}$ This example provides a valuable opportunity for researchers and healthcare providers to reflect on the potential implications of intelligent-enabled technologies and their impact on clinical practice.

Although intelligent agents may, in their current state, have limited ability to provide treatment decisions, their human-like characteristics and attributes may disrupt and alter the role of health care providers and may cause job loss due to automation. ${ }^{6}$ An evaluation by Frey and Osbourne that examined the susceptibility of jobs to automation found that physicians, nurses, dentists are among a group of healthcare providers that are considered the least likely to be replaced by automation. ${ }^{11}$ On the other hand, healthcare workers such as medical and clinical laboratory technologists are amongst the group of professionals that are very likely to be replaced by automation. ${ }^{11}$ Additionally, though it was suggested that certain jobs are less likely to be replaced, it does not necessarily mean that the nature of the job will remain the same. It has been suggested that intelligent agents will not serve as a substitute or replacement for professionals, but will instead increase professionals' effectiveness. ${ }^{12}$ What may this mean? For example, how will the introduction of intelligent agents in the clinical setting modify the scope of practice and responsibilities of physicians and nurses? How will these intelligent agent-enabled technologies influence clinical practice? If clinical decisions such as treatment plans fall into the hands of intelligent agents, who will be responsible for patients' care plan?

Consideration must be given to the diffusion of intelligent agents into the healthcare setting and their impact on clinical decision making as they become more sophisticated and powerful. Since intelligent agents possess the capabilities to problem solve and provide solutions based on its surrounding environment and stimuli (as demonstrated by IBM Watson), they may assist with, and perhaps even intervene in, health care providers' ability to formulate clinical decisions. As such, the intersection of intelligent agents and clinical decision making requires significant discussion and examination. The example of IBM Watson reinforces the importance of autonomous clinical decision making by practitioners to identify inappropriate treatment plans provided by Watson. Indeed, the ability to possess sound clinical decision making has been considered to be one of the highest attributes for health care providers. $^{13}$

Clinical decision making has been defined as "a contextual, continuous, and evolving process, where data are gathered, interpreted, and evaluated in order to select an evidence-based choice of action." ${ }^{14}$ Sources of information that heath care providers may consider during their clinical decision making process include personal knowledge and experience, scientific evidence, patients' health history, and providers' clinical judgement (including physicians, registered nurses, and other members of allied health).$^{15}$ Several theoretical frameworks and perspectives may provide preliminary insights regarding the potential effects of the integration of intelligent agents into clinical decision making. For example, the Three Stage Decision Making Model by Fellows suggests that individuals seek options prior to an evaluation and subsequently formulate a decision. ${ }^{16}$ In addition, the Shared Decision-Making Model for Clinical Practice by Elwyn proposed that decision support elements are used by either patients or clinicians prior to forming a decision. ${ }^{17}$ These two models have provided an opportunity to explore the ability of intelligent agents to impact clinical decision making via providing additional options and recommendations for health care providers based on their unique ability to semi- or fullyautonomously execute planning and problem solving. The provision of these options could be seen in both positive and negative lights. 
For example, increased options generated by the agents may provide a more comprehensive approach to identifying treatment options for patients, yet the overabundance of options may also lead to decision fatigue, resulting in less favorable decisions. Given the wide range of factors and complexities that currently underpins health care providers' clinical decision making, a closer examination of the potential impact of intelligent agents is warranted.

Though not comprehensive in nature, the purpose of this commentary was to provide insight and to generate discussion regarding the integration of intelligent agents and how it may influence health care providers' decision making process. It is important to recognize that although there are many foreseeable benefits associated with intelligent agents in the clinical setting, further evidence is required to support their full and systematic implementation. Additionally, much of intelligent agents' current capabilities rely on predictions based on past data and information. How does this compare with health care providers' clinical decision making? Do health care providers currently operate based on a prediction model or an evidence-based decision making process? Given the potentially limitless opportunities for research and development for intelligent agents, clinical decision making is only one of the many avenues that researchers may pursue and investigate. Further research regarding the efficacy of intelligent agents may generate evidence to support their adoption and integration in the healthcare setting to assist healthcare practitioners and their provision of care.

\section{REFERENCES}

1. Tecuci G. Artificial intelligence. Wiley Interdiscip Rev Comput Stat. 2012 Dec;4(2):168-80. https://doi.org/10.1002/wics.200

2. Russell SJ, Norvig P. Artificial intelligence: A modern approach. 3rd ed. Upper Saddle River, NJ: Prentice Hall; 2010. 1132 p.

3. Isern, D, Moreno A. A systematic literature review of agents applied in healthcare. J Med Syst. 2016 Nov;40(2),1-14. https://doi.org/10.1007/ s10916-015-0376-2

4. McMurray J, Strudwick G, Forchuk C, et al. The importance of trust in the adoption and use of intelligent assistive technology by older adults to support aging in place: scoping review protocol. JMIR Res Protoc. 2017 Nov;6(11):e218. https://doi.org/10.2196/resprot.8772

5. Chen Y, Elenee Argentinis JD, Weber G. IBM Watson: how cognitive computing can be applied to big data challenges in life sciences research. Clin Ther. 2016 Apr;38(4):688-701. https://doi.org/10.1016/j. clinthera.2015.12.001

6. Vogel L. Plan needed to capitalize on robots, AI in health care. CMAJ. 2017 Feb;189(8):e329-30. https://doi.org/doi:10.1503/cmaj.1095395

7. Doyle-Lindrud S. Watson will see you now: A supercomputer to help clinicians make informed treatment decisions. Clin J Oncol Nurs. 2015 Feb;19(1):31-2. https://doi.org/10.1188/15.CJON.31-32

8. Kris M. Interview: progress and promise in the therapy of lung cancers. Lung Cancer Manag. 2014 Feb;3(1):17-21. https://doi. org/10.2217/lmt.13.78

9. Schilling RB. What hospitals can learn from IBM Watson health's challenges. Appl Radiol. 2017 Sep;46(9):8.

10. Bernal N. IBM Watson AI criticised after giving 'unsafe' cancer treatment advice. The Telegraph. 2018 July 27 [cited 2018 Aug 8]. https://www.telegraph.co.uk/technology/2018/07/27/ibm-watson-aicriticised-giving-unsafe-cancer-treatment-advice/
11. Frey CB, Osborne MA. The future of employment: how susceptible are jobs to computerisation? Technol Forecast Soc Change. 2017 Sep;114:254-80. https://doi.org/10.1016/j.techfore.2016.08.019

12. O'Neill C. Is AI a threat or benefit to health workers? CMAJ. 2017 May;189(20):e732. https://doi.org/10.1503/cmaj.1095428

13. Croskerry P, Norman G. Overconfidence in clinical decision making. Am J Med. 2008 May;121(5): s24-9. https://doi.org/10.1016/j. amjmed.2008.02.001

14. Tiffen J, Corbridge SJ, Slimmer L. Enhancing clinical decision making: development of a contiguous definition and conceptual framework. J Prof Nurs. 2014 Sep;30(5):399-405. https://doi.org/10.1016/j. profnurs.2014.01.006

15. Murphy J. Decision making and the new technologies in clinical practice. Ir Med J. 2017 Jun;110(6):577.

16. Fellows LK. The cognitive neuroscience of human decision making: a review and conceptual framework. Behav Cogn Neurosci Rev. 2004 Sep;3(3):159-72. https://doi.org/10.1177/1534582304273251

17. Elwyn G, Frosch D, Thomson R, et al. Shared decision making: a model for clinical practice. J Gen Intern Med. 2012 Oct;27(10):1361-7. https:// doi.org/10.1007/s11606-012-2077-6 